\section{Regulation by Insulin of Myocardial Glucose and Fatty Acid Metabolism in the Conscious Dog}

\author{
Eugene J. Barrett, Ronald G. Schwartz, Charles K. Francis, \\ and Barry L. Zaret \\ Department of Medicine, Yale University School of Medicine, \\ New Haven, Connecticut 06510
}

bstract. In vivo small doses of insulin inhibit lipolysis, lower plasma FFA, and stimulate glucose disposal. Lowering of plasma FFA, either in the absence of a change in insulin or during combined hyperglycemia and hyperinsulinemia, promotes glucose uptake by heart muscle in vivo. In the isolated perfused heart, large doses of insulin directly stimulate heart glucose uptake. To assess the effect of physiological elevations of plasma insulin upon myocardial glucose and FFA uptake in vivo independent of changes in plasma substrate concentration, we measured arterial and coronary sinus concentrations of glucose, lactate, and FFA, and coronary blood flow in conscious dogs during a $30 \mathrm{~min}$ basal and a $2 \mathrm{~h}$ experimental period employing three protocols: (a) euglycemic hyperinsulinemia (insulin clamp, $n=5$ ), (b) euglycemic hyperinsulinemia with FFA replacement $(n=5),(c)$ hyperglycemic euinsulinemia (hyperglycemic clamp with somatostatin, $n=5$ ).

In group 1, hyperinsulinemia (insulin $=73 \pm 13 \mu \mathrm{U} /$ $\mathrm{ml})$ stimulated heart glucose uptake (7.3 \pm 4.4 vs. $28.2 \pm 2.8$ $\mu \mathrm{mol} / \mathrm{min}, P<0.002$ ), lowered plasma FFA levels by $80 \%(P<0.05)$, and decreased heart FFA uptake $(28.4 \pm 4$ vs. $1.5 \pm 0.9, P<0.01)$. When the fall in plasma FFA was prevented by FFA infusion (group 2), hyperinsulinemia $(86 \pm 10 \mu \mathrm{U} / \mathrm{ml})$ provoked a lesser $(P<0.05)$ stimulation of glucose uptake $(\Delta=8.2 \pm 4.2 \mu \mathrm{mol} / \mathrm{min})$ than in group 1 , and there was no significant change in FFA uptake $(25.3 \pm 16$ vs. $16.5 \pm 4)$. Hyperglycemia

Address correspondence to Dr. Barrett, Endocrinology Unit, Department of Medicine, Yale University School of Medicine, 333 Cedar St., New Haven, CT, 06510.

Received for publication 20 December 1983 and in revised form 3 May 1984

J. Clin. Invest.

(C) The American Society for Clinical Investigation, Inc. 0021-9738/84/09/1073/07 \$1.00

Volume 74, September 1984, 1073-1079 (plasma glucose $=186 \pm 8 \mathrm{mg} / 100 \mathrm{ml}$ ) during somatostatin infusion resulted in only a small rise in plasma insulin $(\Delta=12 \pm 7 \mu \mathrm{U} / \mathrm{ml})$, and although plasma FFA tended to decline, heart glucose uptake did not rise significantly $(\Delta=5.5 \pm 3.2 \mu \mathrm{mol} / \mathrm{min}, P=\mathrm{NS})$. There was no significant change in coronary blood flow during any of the three study protocols.

We conclude that, in the dog, insulin at physiologic concentrations: $(a)$ stimulates heart glucose uptake, both directly and by suppressing the plasma FFA concentration, and $(b)$ does not alter coronary blood flow. Hyperglycemia per se has little effect on heart glucose uptake.

\section{Introduction}

In the postabsorptive state, cardiac muscle, like skeletal muscle, utilizes FFA as the principal oxidative fuel (1). Circulating glucose and heart glycogen furnish additional fuels, which may be of particular importance when oxygen availability is limited, as may occur in acute or chronic myocardial ischemia (2). In healthy man, the arterial fatty acid concentration uppears to be the principal determinant of heart fatty acid extraction (3). Two decades ago, Randle et al. $(4,5)$ observed that in the perfused rat heart, fatty acids inhibit the oxidation of glucose and proposed that a "glucose-fatty acid cycle" existed whereby fat oxidation inhibited the flux of carbohydrates through the glycolytic pathway. This inhibition of carbohydrate oxidation persisted even in the presence of pharmacologic doses of insulin. In contrast, in the absence of exogenous fatty acids, high concentrations of insulin greatly stimulate heart glucose uptake, glycogen synthesis, and carbohydrate oxidation (6). While abundant work in the isolated perfused heart has demonstrated that insulin at high concentrations stimulates the uptake and metabolism of glucose $(7-10)$, evidence that insulin at physiologic concentrations regulates heart glucose uptake in vivo is indirect (11). Furthermore, since in vivo insulin lowers plasma FFA levels by inhibiting lipolysis in adipose tissue (12), assessment of insulin's direct role in regulating heart glucose metabolism requires simultaneous estimation of heart fatty acid exchange. Clarification of the 
mechanisms of myocardial metabolic control are of importance with respect to further understanding of myocardial ischemia and its therapy. In addition, changes in arterial concentration of insulin, glucose, or FFA might influence myocardial extraction of fuel substrates as observed in vivo using radioactive glucose and positron-computed tomographic imaging.

In the present study, we used the insulin clamp technique (13) in combination with arterial and coronary sinus blood sampling in the conscious dog to study the effect of physiologic changes in plasma insulin on the myocardial extraction of glucose, FFA, and lactate. Circulating concentrations of FFA and glucose were altered independently by infusing heparin with triglyceride emulsion and adjusting the glucose infusion rate during the insulin clamp. The effect of hyperglycemia, independent of alterations in plasma insulin, on heart fuel substrate exchange was also assessed using the glucose clamp technique (13). High doses of insulin have been reported to increase coronary blood flow in conscious euglycemic dogs (14). Insulin-induced changes in coronary flow could alter the net myocardial uptake or release of substrate, despite a constant arterial-coronary sinus substrate concentration gradient. Therefore, in addition to measurements of substrate extraction, serial thermodilution estimates of coronary blood flow were obtained during the course of these experiments.

\section{Methods}

Animal preparation. 24-48 h before study, adult mongrel dogs of either sex weighing between 18 and $31 \mathrm{~kg}$ (mean $=24 \pm 2 \mathrm{~kg}$ ) were anesthetized with barbital (Surital). Under aseptic conditions, an incision was made in the right side of the neck and the carotid artery and jugular vein were isolated. A heparin-filled silastic catheter (1.25 mm interior diam., Dow Corning, Midland, MI) was placed in each vessel, and the extravascular ends occluded and fixed in the subcutaneous tissue. The overlying skin was then sutured closed. On the day of study, after an overnight fast, the awake animal was gently restrained on a fluoroscopy table in a left lateral decubitus position. Following administration of lidocaine locally, the sutures were removed from the neck, and the free ends of the catheters exposed. The arterial catheter was used for obtaining arterial blood samples and for monitoring blood pressure. The second silastic catheter was used as an introducer for a triple thermistor thermodilution catheter (Webster Laboratories Inc., Altadena, CA) which was then positioned in the main portion of the coronary sinus under fluoroscopic guidance. A \#7F Sones catheter was also advanced into the coronary sinus and used for sampling of coronary sinus blood. The thermodilution and sampling catheters both remained in the coronary sinus throughout the study and the catheters' position was confirmed fluoroscopically at the end of the study. Normal saline was infused through the thermodilution catheter at a rate of 32 $\mathrm{ml} / \mathrm{min}$ for 30-60 $\mathrm{s}$ for each determination of coronary flow. An 18gauge polyethylene catheter was placed percutaneously in the right saphenous vein and used for infusion of hormones and substrates. Patency of all catheters was maintained by a slow infusion of normal saline. Position of the coronary sinus catheters was confirmed at the beginning and end of each experiment by fluoroscopy and measurement of oxygen saturation.

Experimental protocols. Coronary sinus blood flow and arterial and coronary sinus concentrations of glucose, FFA, and lactate were measured every $10 \mathrm{~min}$ during a 30 -min basal period and every 20 min during the 2-h experimental period. Five dogs were studied in each of three experimental protocols. In group 1, the euglycemic insulin clamp was employed. After obtaining basal samples, plasma insulin was raised $\sim 60 \mu \mathrm{U} / \mathrm{ml}$ above fasting values using a primed ( 2 $\mathrm{mU} / \mathrm{min} \cdot \mathrm{kg} \times 10 \mathrm{~min})$ continuous $(1 \mathrm{mU} / \mathrm{min} \cdot \mathrm{kg} \times 110 \mathrm{~min})$ infusion of regular insulin (Eli Lilly Co., Indianapolis, IN). Plasma glucose was maintained constant at basal values by measuring plasma glucose every 5 min and adjusting the rate of infusion of a $20 \%$ dextrose solution as previously described (13).

In group 2 , plasma insulin was again raised by $\sim 60 \mu \mathrm{U} / \mathrm{ml}$ above basal and plasma glucose maintained at basal levels by a variable rate glucose infusion as in group 1 . In addition, a soybean triglyceride emulsion (Intralipid 20\%, Cutter Laboratories, Berkeley, CA) was infused $(0.5 \mathrm{ml} / \mathrm{min})$ with sodium heparin $(20 \mathrm{U} / \mathrm{min})$ to prevent the decline in plasma FFA caused by hyperinsulinemia.

In group 3 , somatostatin was infused $(500 \mu \mathrm{g} / \mathrm{h})$ to inhibit endogenous insulin secretion, while plasma glucose was acutely raised and maintained constant at $80 \mathrm{mg} / 100 \mathrm{ml}$ above basal using a variable rate glucose infusion ( $+80 \mathrm{mg} / 100 \mathrm{ml}$ glucose clamp) as previously described (13).

Chemical determinations. Plasma glucose was measured using the glucose oxidase method and a Beckman glucose analyzer (Beckman Instruments, Inc., Palo Alto, CA). FFA were measured by a modification of the procedure of Dole (15); blood lactate and plasma insulin were assayed as described previously $(16,17)$. $\mathrm{Blood} \mathrm{pH}, \mathrm{PO}_{2}$ and $\mathrm{PCO}_{2}$ were measured using an Ilford (Micro 13) blood gas analyzer (Instrumentation Laboratories, Lexington, MA).

Calculations. Coronary sinus blood flow was estimated from the thermodilution curves as described by Ganz et al. (18) and validated by Pepine et al. (19). Four separate estimates of coronary flow were obtained during the basal period and two estimates were made every $20 \mathrm{~min}$ thereafter, immediately prior to obtaining coronary sinus blood samples. Myocardial uptake or release of substrate was calculated from the product of the arterial-coronary sinus substrate concentration difference and the simultaneously obtained coronary flow. Because of differences in their intravascular distribution, for glucose and FFA fluxes plasma concentrations and coronary plasma flows [coronary blood flow $\times$ (1-hematocrit)] were used, while for lactate whole blood concentrations and blood flows were employed. The substrate extraction ratio at any time was determined by dividing the arterial-coronary sinus substrate concentration difference by the arterial substrate concentration.

Statistical analysis of the change in myocardial substrate exchange over time and comparison among experimental protocols were performed using either paired or unpaired $t$ test or analysis of variance with a repeated measures design (20). Data are expressed as mean \pm SEM.

\section{Results}

During the $30 \mathrm{~min}$ basal period, the myocardium of all dogs took up fatty acids $(21 \pm 5 \mu \mathrm{mol} / \mathrm{min})$. In the basal state, the mean extraction ratio for FFA was $30 \pm 3 \%$ and was independent of arterial FFA concentration. However, there was a strong correlation between the arterial FFA concentration and heart FFA uptake $(r=0.88, P<0.01)$. There was also a significant uptake of glucose $(10.6 \pm 3.5 \mu \mathrm{mol} / \mathrm{min})$, but the extraction ratio $(2.2 \pm 0.9 \%)$ was much less than for FFA. The 
Table I. Myocardial Uptake of Glucose and FFA During the 30-Min Basal Period and During the Last Hour of the Experimental Period

\begin{tabular}{lcc}
\hline & $\begin{array}{l}\text { Glucose } \\
\text { uptake }\end{array}$ & FFA uptake \\
\hline & $\mu \mathrm{mol} / \mathrm{min}$ & $\mu \mathrm{mol} / \mathrm{min}$ \\
Group I & & \\
Basal & $7.3 \pm 4.4$ & $28.4 \pm 4.0$ \\
$60-120 \mathrm{~min}$ & $28.2 \pm 2.8^{*}$ & $1.5 \pm 0.9^{*}$ \\
$\Delta$ & $20.9 \pm 2.8$ & $-26.9 \pm 4.5$ \\
Group II & & \\
Basal & $15.2 \pm 5.1$ & $25.3 \pm 16$ \\
$60-120 \mathrm{~min}$ & $23.4 \pm 3.3$ & $16.5 \pm 4$ \\
$\Delta$ & $8.2 \pm 4.2 \ddagger$ & $-8.8 \pm 14$ \\
Group III & & \\
Basal & $7.9 \pm 7.7$ & $13.5 \pm 4.9$ \\
$60-120 \mathrm{~min}$ & $12.4 \pm 9.8$ & $3.7 \pm 2.2$ \\
$\Delta$ & $4.5 \pm 2.8 \S$ & $-9.8 \pm 6$ \\
\hline
\end{tabular}

All values are mean \pm SEM.

* Denotes uptake in 40-100 min period which was significantly different $(P<0.01)$ than basal uptake within that group.

$\ddagger \S$ Denotes an increment in glucose uptake in groups II and III which were significantly $(P<0.05$ and $P<0.02$, respectively) less than the increment in glucose uptake in group $I$.

basal rates of glucose and FFA uptake by each of the three groups of dogs are given in Table I. During the basal period, there was also a net uptake of lactate by the heart $(18.6 \pm 10.6$ $\mu \mathrm{mol} / \mathrm{min}$ ), and a linear correlation existed between lactate uptake and blood lactate concentration $(r=0.74, P<0.01)$.

The partial pressure of $\mathrm{O}_{2}$ in coronary sinus blood averaged $13 \pm 4 \mathrm{mmHg}$, and the $\mathrm{pH}$ of coronary sinus blood was consistently lower $(\Delta \mathrm{pH}=0.045 \pm 0.004, P<0.001)$ and $\mathrm{PCO}_{2}$ consistently higher $\left(\Delta \mathrm{PCO}_{2}=8.8 \pm 1.4 \mathrm{mmHg}, P<0.01\right)$ than arterial blood during the $30 \mathrm{~min}$ basal period (Table II). Mean basal coronary blood flow averaged $114 \pm 12 \mathrm{ml} / \mathrm{min}$. There were no differences in these parameters among the three groups of dogs.

Results of the euglycemic insulin clamp (group 1) are illustrated in Fig. 1. The plasma insulin concentration rose from basal levels of $10 \pm 2 \mu \mathrm{U} / \mathrm{ml}$ to a mean stimulated value of $73 \pm 13 \mu \mathrm{U} / \mathrm{ml}$ and was maintained constant throughout the study. Arterial glucose was maintained at euglycemic levels. Coronary sinus blood flow was unchanged during the insulin infusion. The arterial-coronary sinus (a-cs) concentration differences for glucose increased following the rise in plasma insulin concentration, while the plasma FFA concentration and the a-cs concentration difference for FFA declined abruptly after raising plasma insulin. Arterial blood lactate concentration during the last hour of study was significantly greater than during the basal period $(450 \pm 80$ vs. $730 \pm 170 \mu \mathrm{mol} / \mathrm{liter}, P$ $<0.05$ ). Heart lactate uptake increased in each of five dogs, but the mean increment in lactate uptake was not statistically significant. As shown in Table II, euglycemic hyperinsulinemia led to a significant stimulation of myocardial glucose uptake $(\Delta=21 \pm 3 \mu \mathrm{mol} / \mathrm{min})$ and a marked depression in myocardial fatty acid removal $(\Delta=-27 \pm 5 \mu \mathrm{mol} / \mathrm{min})$.

In these animals, we observed that the rate of total body glucose utilization, or $M$, was $11.5 \pm 0.4 \mathrm{mg} / \mathrm{min} \cdot \mathrm{kg}$ body weight. The value $M$ during any time interval was estimated from the measured rate of infusion of exogenous glucose during that time interval and corrected for any change in the filling of body glucose space (13).

The data from group 2 dogs are shown in Fig. 2 and Table I. When the plasma insulin concentration was raised from $26 \pm 5$ to $86 \pm 10 \mu \mathrm{U} / \mathrm{ml}$, and plasma glucose maintained at euglycemic levels, infusion of intralipid with heparin (group 2) prevented the decline in FFA seen in group 1. Neither

Table II. Arterial and Coronary Sinus $p H, \mathrm{PO}_{2}$, and $\mathrm{PCO}_{2}$ During the Basal Period and the Last 20 Min of the Experimental Period

\begin{tabular}{|c|c|c|c|c|c|c|}
\hline & \multicolumn{2}{|l|}{$\mathrm{pH}$} & \multicolumn{2}{|c|}{$\mathrm{PO}_{2}(m m H g)$} & \multicolumn{2}{|c|}{$\mathrm{PCO}_{2}(m m H g)$} \\
\hline & Basal & $100-120 \mathrm{~min}$ & Basal & $100-120 \mathrm{~min}$ & Basal & $100-120 \mathrm{~min}$ \\
\hline \multicolumn{7}{|l|}{ Group I } \\
\hline Artery & $7.53 \pm 0.03$ & $7.54 \pm 0.03$ & $66 \pm 7$ & $72 \pm 5$ & $28 \pm 4$ & $26 \pm 3$ \\
\hline Coronary sinus & $7.49 \pm 0.03$ & $7.49 \pm 0.03$ & $11 \pm 1$ & $14 \pm 2$ & $37 \pm 4$ & $36 \pm 4$ \\
\hline \multicolumn{7}{|l|}{ Group II } \\
\hline Artery & $7.51 \pm 0.02$ & $7.49 \pm 0.02$ & $77 \pm 5$ & $70 \pm 4$ & $37 \pm 2$ & $36 \pm 2$ \\
\hline Coronary sinus & $7.45 \pm 0.02$ & $7.45 \pm 0.02$ & $10 \pm 2$ & $12 \pm 2$ & $45 \pm 1$ & $50 \pm 6$ \\
\hline \multicolumn{7}{|l|}{ Group III } \\
\hline Artery & $7.49 \pm 0.03$ & $7.46 \pm 0.03$ & $83 \pm 9$ & $77 \pm 5$ & $35 \pm 4$ & $34 \pm 1$ \\
\hline Coronary sinus & $7.44 \pm 0.03$ & $7.41 \pm 0.03$ & $17 \pm 4$ & $11 \pm 4$ & $44 \pm 5$ & $47 \pm 2$ \\
\hline
\end{tabular}



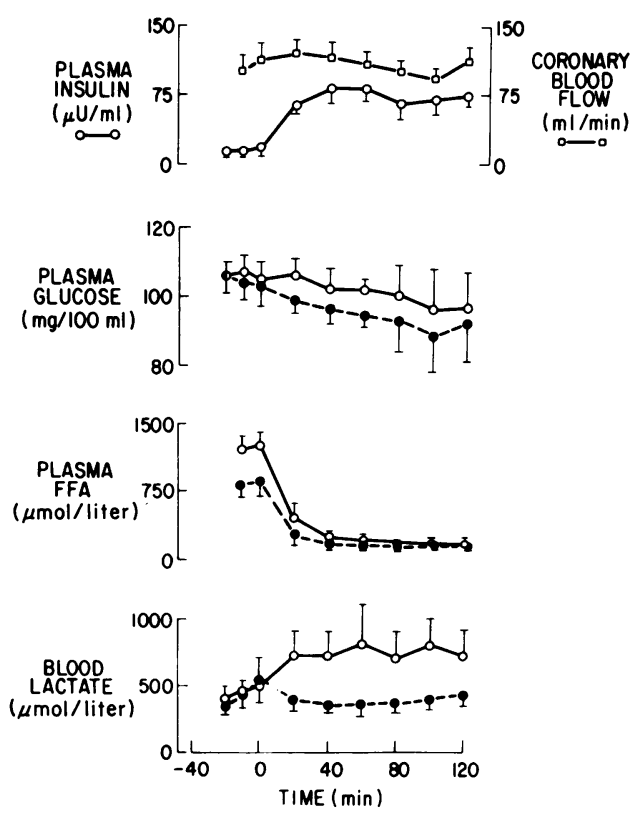

Figure 1. The upper panel illustrates the plasma insulin concentration (o) and the coronary blood flow ( $\square$ ) during the basal period and during the 120-min insulin clamp in five dogs studied in protocol 1. The second, third, and fourth panels show the arterial (O) and coronary sinus $(\bullet)$ concentrations of glucose, FFA, and lactate during the basal and experimental periods. Error bars indicate \pm 1 SEM.
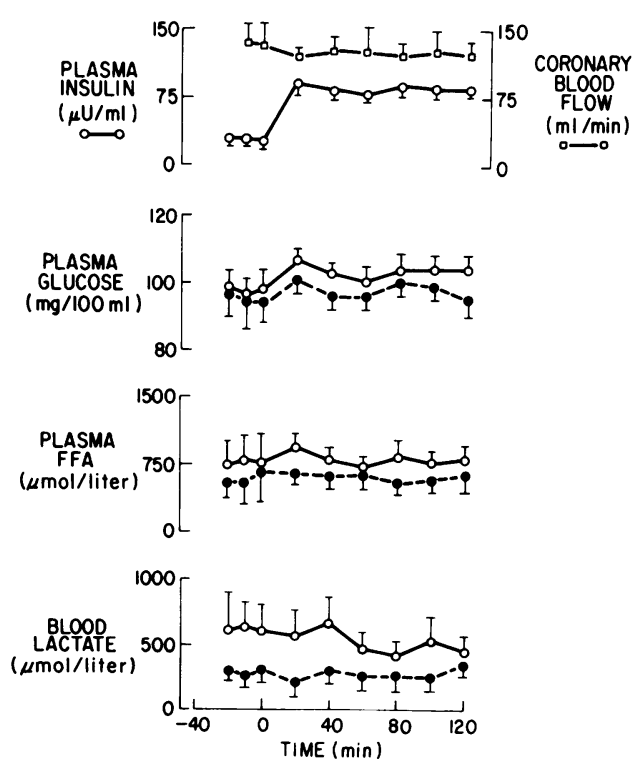

Figure 2. Illustrated are the plasma concentration, coronary blood flow, and arterial and coronary sinus concentrations for glucose, FFA, and lactate in the five dogs studied in protocol 2. Symbols are as designated in Fig. 1. myocardial FFA uptake, FFA extraction ratio, or lactate uptake changed significantly (Fig. 1). Coronary sinus blood flow was again unchanged during the $2 \mathrm{~h}$ of hyperinsulinemia. The higher mean basal myocardial glucose balance in group 2 dogs is attributable to one dog with a basal heart uptake of 33 $\mu \mathrm{mol} / \mathrm{min}$. Hyperinsulinemia did not stimulate glucose uptake in this animal, but significantly increased glucose uptake $(10.7 \pm 3$ vs. $22 \pm 4 \mu \mathrm{mol} / \mathrm{min}, P<0.025)$ in the remaining four dogs.

During the euglycemic clamp, $M$ averaged $6.2 \pm 0.5 \mathrm{mg} /$ $\mathrm{min} \cdot \mathrm{kg}$ in the five dogs in group 2 . This was significantly less $(P<0.001)$ than the corresponding measure $(11.5 \pm 0.4 \mathrm{mg} /$ $\mathrm{min} \cdot \mathrm{kg}$ ) obtained in group 1.

During the hyperglycemic clamp (group III), the steady state plasma glucose concentration averaged $188 \pm 10 \mathrm{mg} / 100$ $\mathrm{ml}$ (Fig. 3). Somatostatin infusion inhibited the rise in plasma insulin that would normally accompany marked hyperglycemia, and the mean plasma insulin during the period of hyperglycemia averaged only $12 \pm 7 \mu \mathrm{U} / \mathrm{ml}$ above basal $(P=N S)$. Coronary sinus blood flow did not change from basal (Fig. 3). There was no significant change in myocardial glucose uptake ( $8 \pm 8$ vs. $12 \pm 10 \mu \mathrm{mol} / \mathrm{min})$ or in heart FFA uptake $(\Delta=-9.8 \pm 6 \mu \mathrm{mol} /$ min) (Table I).

Total body glucose utilization in these dogs averaged $8.6 \pm 1.4 \mathrm{mg} / \mathrm{min} \cdot \mathrm{kg}$. In these hyperglycemic animals, this estimate of total glucose utilization includes urinary glucose losses which could not be accurately estimated in these con-

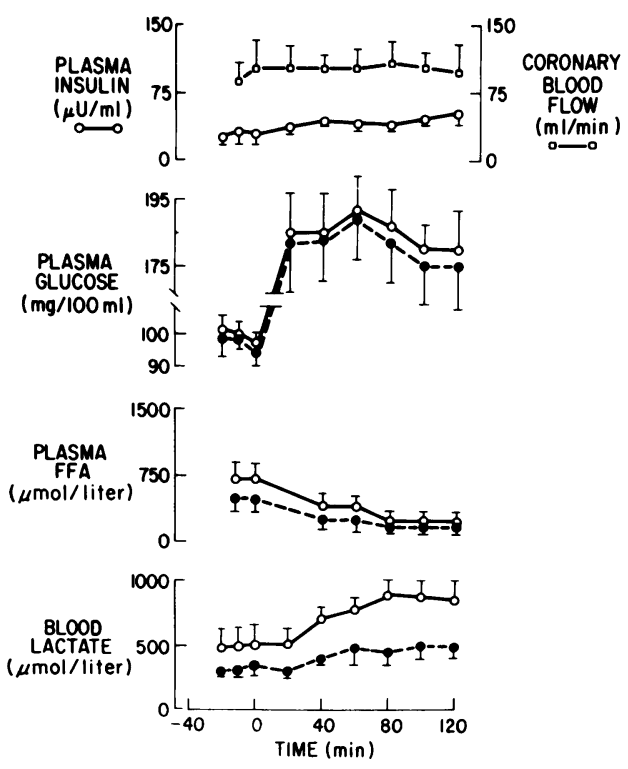

Figure 3. Coronary blood flow and plasma concentrations of insulin, glucose, FFA, and blood lactate in the artery and coronary sinus samples are shown for the five dogs studied using protocol 3. See Fig. 1 for explanation of symbols. 
scious dogs. Therefore, the $8.6 \pm 1.4 \mathrm{mg} / \mathrm{min} \cdot \mathrm{kg}$ could be regarded as an upper limit to the rate of body glucose utilization in these animals. Table I compares the stimulation of glucose uptake (designated $\Delta$ ) above postabsorptive rates, which occurred over the last hour of each study protocol. It is apparent that while myocardial glucose uptake increased in each group, the magnitude of stimulated glucose uptake is greatest when a rise in plasma insulin is accompanied by a decline in plasma FFA (group I). The increment in glucose uptake in group I dogs $(20.9 \pm 3.0 \mu \mathrm{mol} / \mathrm{min}, n=5)$ was significantly greater than that in group II (lipid-infused) dogs $(n=5)$ as a whole $(\Delta=8.2 \pm 4.2 \mu \mathrm{mol} / \mathrm{min}, P<0.05)$. This significance persists even when the single insulin unresponsive dog in group II is excluded $(\Delta=11.7 \pm 3.3 \mu \mathrm{mol} / \mathrm{min}, P$ $<0.05, n=4$ ).

\section{Discussion}

Results of the current study demonstrate that elevation of plasma insulin within the physiologic range has a clear stimulating effect on myocardial glucose uptake in the awake dog. This stimulatory action of insulin occurs in the absence of a change in plasma glucose or coronary blood flow and on average, results in a fourfold enhancement in heart glucose extraction.

The insulin-induced stimulation of myocardial glucose removal appears to occur both by direct facilitation of glucose extraction and indirectly by insulin-induced inhibition of lipolysis resulting in depression of circulating fatty acid levels. Our observation that FFA blunt the stimulatory effect of insulin on myocardial glucose uptake is in accord with the early suggestion by Randle et al. (5) that FFA inhibit glucose oxidation through inhibition of phosphofructokinase, pyruvate dehydrogenase, or both. In perfused heart, Williamson (21) subsequently demonstrated that acetate could similarly inhibit insulin-stimulated glucose oxidation. Acetate, like fatty acids, appeared to exert its major inhibitory activity via an increased cytosolic citrate concentration and inhibition of phosphofructokinase. In considering the possible site of the FFA-induced inhibition of myocardial glucose uptake in the current study, we observed a linear correlation between heart lactate uptake and arterial lactate concentration during the last hour of the three study protocols $(r=0.81, P<0.01)$. Furthermore, regardless of whether the arterial FFA concentrations were normal (group II) or suppressed (group I), the myocardial lactate extraction ratios were similar $(37 \pm 15$ and $40 \pm 12 \%$, respectively). This would suggest that in vivo, under physiological conditions, the primary site of FFA inhibition of heart glucose uptake occurs before pyruvate dehydrogenase and may involve phosphofructokinase or glucose transport per se (10).

It bears emphasis that the FFA levels seen in group II during intralipid and heparin infusion were not elevated above postabsorptive levels. This finding implies that FFA within the physiologic range are a major modifier of insulin-induced myocardial glucose uptake. In addition, we observed that in the animals in group II, total body glucose utilization, $M$, was significantly less than the glucose metabolic rate seen in group I dogs. This would be expected if in the dog, the infused fatty acids antagonized the insulin-induced stimulation of glucose uptake by skeletal as well as cardiac muscle (skeletal muscle is the principal site of glucose disposal during euglycemic hyperinsulinemia) (22). Indeed, recent studies in man have shown that under experimental conditions similar to those employed here, elevation of the plasma FFA concentration significantly blunts insulin-stimulated glucose uptake by skeletal muscle $(23,24)$. Again, Randle et al. (5) have previously shown that in the isolated hemidiaphragm, FFA can inhibit insulin-stimulated glucose uptake and oxidation. However, studies in sartorius muscle (25), perfused hindquarters (26), and rat diaphragm (27) failed to show any effect of FFA upon glucose uptake. The more recent work of Rennie and Holloszy (28) using perfused hindquarter suggest that the inhibitory effect of oleate on glucose utilization is clearly demonstrable only when the skeletal muscle is very well oxygenated. Differences in tissue oxygenation may account for some of the apparent lack of effect of fatty acids on glucose utilization in earlier studies.

Thus, while multiple investigators have described an inhibitory effect of FFA on glucose uptake and oxidation in isolated perfused hearts, in vitro studies in skeletal muscle have yielded conflicting results. In addition to the adequacy of oxygen supply, multiple factors, including imposed work load (10), insulin concentration (5), and the rate of generation of FFA within the isolated muscle preparation, can each affect muscle carbohydrate handling; therefore, it is perhaps not surprising that a consistent effect of FFA (or ketones) on carbohydrate utilization in isolated skeletal muscle preparations has not emerged. Results of the current study in conscious animals strongly supports the hypothesis that in vivo and under physiologic conditions, the circulating concentration of fatty acids is an important mediator of glucose metabolism in both cardiac and skeletal muscle, even when the plasma insulin concentration is significantly elevated.

Lassers and colleagues (29) have clearly demonstrated that in postabsorptive man, nicotinic acid lowers the plasma FFA and increases the myocardial uptake of glucose, lactate, and pyruvate. This nicotinate-enhanced glucose uptake occurred in the absence of any rise in plasma insulin (30). The respiratory quotient also increased, indicating a shift in myocardial metabolism to aerobic glycolysis. Kaijser and his associates (31) have also examined carbohydrate extraction by the human heart during exercise or glucose infusion. During exercise, plasma FFA rose markedly while insulin and glucose declined, and heart uptake of FFA but not glucose increased (31). In contrast, during glucose infusion, plasma glucose and insulin and myocardial glucose uptake rose while plasma levels and myocardial removal of FFA declined. In these latter studies, unlike our results reported here, the plasma glucose, insulin, 
and FFA each changed. Therefore, it was not possible to assess separately their effects upon myocardial substrate exchange. Results of the current study are entirely consistent with the hypothesis that plasma FFA concentrations are an important modulator of cardiac substrate exchange during fasting and when plasma insulin is raised within the physiologic range. Our observation that in four of the dogs in group II, myocardial glucose uptake rose during insulin infusion even though the decline in plasma free fatty acids was prevented by lipid infusion suggests that insulin has direct stimulatory effects on heart glucose metabolism. Such a direct effect of physiologic concentrations of insulin on heart glucose uptake may be a reflection of the well-known stimulatory effect of pharmacologic doses of insulin on glucose metabolism in the isolated perfused heart. In contrast to the important role of alterations of plasma FFA and of plasma insulin on heart metabolism, elevations of plasma glucose in the absence of a significant rise in insulin (group III) cause little change in cardiac substrate exchange.

It should be noted that in dogs receiving intravenous insulin (groups I and II), no change in coronary blood flow occurred despite an increment in plasma insulin of $\sim 60 \mu \mathrm{U} /$ $\mathrm{ml}$. These data stand in contrast to the work of Liang et al. (14). They recently reported that insulin, given in doses four to eight times higher than those employed in our studies, caused myocardial blood flow, measured by the radioactive microsphere technique, to increase by $\sim 40 \%$ if blood glucose was allowed to fall. Maintenance of euglycemia resulted in a lesser $(\sim 30 \%)$ but still significant increase in myocardial blood flow. Beta adrenergic blockade prevented the insulin-induced rise in coronary flow. The higher insulin infusion rates employed in that study likely account for the increased flow observed, since insulin stimulation of sympathetic discharge appears to be dose-dependent (32). Liang et al. (14) also noted increases in heart rate, cardiac output, and left ventricular $\mathrm{dP} / \mathrm{dt}$ in response to insulin. Propranolol effectively blocked each of the changes in cardiac function, which accompanied insulin infusion in both euglycemic and hypoglycemic animals. These observations again suggest that insulin's effect was indirect and mediated via beta adrenergic stimulation. We observed no change in heart rate, arterial blood pressure, or pulse pressure during protocols 1 and 2 when insulin is infused at a rate of the $1 \mathrm{mU} / \mathrm{min}$ per kilogram.

The current study also provides physiologic data which have implications concerning the use of radionuclide imaging to assess myocardial metabolic events. The advent of positron and single photon-computed tomography and the production of radiolabeled carbon- 11 and fluorine- 18 metabolites currently allows direct assessment of regional metabolic function. Studies have involved qualitative as well as quantitative measures of regional myocardial uptake and distribution of fatty acid and glucose analogs, and regional perfusion in experimental animals and man $(33,34)$. Increased regional accumulation of 2-fluoro2-deoxyglucose has been used as a marker of myocardial viability in ischemic portions of the left ventricle where anerobic glucose metabolism has been operative (34). Awareness of the effect of a varying metabolic milieu upon myocardial utilization of these substrates is critical for understanding measurements of altered metabolism under pathologic circumstances.

In summary, findings in the current study support the hypothesis that at physiologic concentrations, plasma insulin is a major regulator of myocardial substrate exchange in the conscious dog. Insulin promotes myocardial carbohydrate metabolism directly by stimulating carbohydrate uptake and indirectly through inhibition of lipolysis. The lowered plasma concentration of FFA mediates the indirect action of insulin, and infusion of FFA significantly blunts insulin stimulation of myocardial carbohydrate removal. Hyperglycemia, in the absence of elevated insulin levels, has little effect on heart glucose uptake. Finally, our results do not support a significant role for insulin at physiological concentrations in the modulation of coronary blood flow.

\section{Acknowledgments}

We wish to thank Frank Pelletier and Ralph Jacob for technical assistance, Dr. R. Hendler for performing the insulin immunoassay, and Mrs. D. Bigelow and N. Canetti for assistance in preparing the manuscript.

This work was supported in part through American Heart Association grant 83-1264. Dr. R. Schwartz is recipient of U. S. Public Health Service fellowship No. HL-06744-01. Dr. E. Barrett is recipient of U. S. Public Health Service Special Emphasis Research Career Award-Diabetes Mellitus:Cardiovascular AM00888.

\section{References}

1. Bing, R. J. 1965. Cardiac metabolism. Physiol. Rev. 45:171202.

2. Liedtke, A. J. 1981. Alterations of carbohydrate and lipid metabolism in the acute ischemic heart. Prog. Cardiovasc. Dis. 23:321336.

3. Wahlquist, M. L., L. Kaijser, B. Lassers, and L. Carlson. 1973. Fatty acid as a determinant of myocardial substrate and oxygen metabolism in man at rest and during prolonged exercise. Acta Med. Scand. 193:89-96.

4. Randle, P., C. Hales, P. Garland, and E. Newsholme. 1963. The glucose fatty-acid cycle. Lancet. I:785-789.

5. Randle, P. J., E. A. Newsholme, and P. B. Garland. 1964. Regulation of glucose uptake by muscle .8. Effects of fatty acids, ketone bodies and pyruvate and of alloxan-diabetes and starvation on the uptake and metabolic rate of glucose in rat heart and diaphragm muscles. Biochem. J. 93:652-665.

6. Randle, P. J., and P. K. Tubbs. 1979. Carbohydrate and fatty acid metabolism. In Handbook of Physiology: the Cardiovascular system I. American Physiology Society, Bethesda, MD. 805-844.

7. Cheung, J. Y., C. Conover, D. M. Regen, C. Whitfield, and H. E. Morgan. 1978. Effect of insulin on kinetics of sugar transport in heart muscle. Am. J. Physiol. 234:E70-E78.

8. Park, C. R., H. E. Morgan, M. J. Henderson, D. M. Regen, E. Cadenas, and R. L. Post. 1961. The regulation of glucose uptake in 
muscle as studied in the perfused rat heart. Recent Prog. Horm. Res. 17:493-529.

9. Fisher, R. B., and D. B. Lindsay. 1956. The action of insulin on the penetration of sugars into the perfused heart. J. Physiol. (Lond.). 131:526-561.

10. Neely, J. R., R. H. Bowman, and H. E. Morgan. 1969. Effects of ventricular pressure development and palmitate on glucose transport. Am. J. Physiol. 216:804-811.

11. Wahlquist, M., L. Kaijser, B. Lassers, H. Low, and L. Carlson. 1973. The role of fatty acid and of hormones in the determination of myocardial carbohydrate metabolism in healthy fasting men. Europ. J. Clin. Invest. 3:57-65.

12. Fain, J. N., V. P. Kovacev, and R. O. Scow. 1966. Antilipolytic effect of insulin in isolated fat cells of the rat. Endocrinology. 78:773778.

13. DeFronzo, R., J. Tobin, and R. Andres. 1979. The glucose clamp technique. Am. J. Physiol. 237:E214-E223.

14. Liang, C., J. Doherty, R. Failace, K. Mackawa, S. Arnold, H. Gavras, and W. Hood. 1982. Insulin infusion in conscious dogs. Effects on systemic and coronary hemodynamics, regional blood flow, and plasma catecholamines. J. Clin. Invest. 69:1321-1336.

15. Novak, M. 1965. Colorometric ultramicro method for the determination of free fatty acids. J. Lipid Res. 6:431-433.

16. Gutman, I., and A. Wahlefeld. 1974. $\mathrm{L}(+)$ lactate determination with lactic dehydrogenase and NAD. Methods Enzymatic Analysis. 3:1464-67.

17. Wise, J. K., R. Hendler, and P. Felig. 1973. Influence of glucocorticoids on glucagon secretion and amino acid concentrations in man. J. Clin. Invest. 52:2774-2784.

18. Ganz, W., K. Tamura, H. Marcus, R. Donoso, S. Yoshido, and $H$. Swan. 1971. Measurement of coronary sinus blood flow by continuous thermodilution in man. Circulation. 44:181-195.

19. Pepine, C., J. Mehta, W. Webster, and W. Nichols. 1978. In vivo validation of a thermodilution method to determine regional left ventricular blood flow in patients with coronary disease. Circulation. 58:795-802.

20. Snedecor, G. W., and W. G. Cochran. 1980. Statistical Methods. Seventh ed. Iowa Univ. Press, Ames, IA. 255-273.

21. Williamson, J. R. 1965. Glycolytic control mechanisms. J. Biol. Chem. 240:2308-2321.

22. DeFronzo, R. A., E. Jacot, E. Jequier, E. Maeder, J. Wahren, and J. P. Felber. 1981. The effects of insulin on the disposal of intravenous glucose. Results from indirect colorimetry and hepatic and femoral venous catheterization. Diabetes. 30:1000-1007.

23. Ferrannini, E., E. J. Barrett, S. Bevilacqua, and R. A. DeFronzo. 1983. Effects of fatty acids on glucose production and utilization in man. J. Clin. Invest. 72:1737-1747.

24. Schalch, P. S., and D. M. Kipnis. 1965. Abnormalities in carbohydrate tolerance associated with elevated plasma nonesterified fatty acids. J. Clin. Invest. 44:2010-2020.

25. Cassens, R. G., R. M. Bocek, and C. H. Beatty. 1969. Effect of octanoate on carbohydrate metabolism in red and white muscles of the rhesus monkey. Am. J. Physiol. 217:715-719.

26. Goodman, M. N., M. Berger, and N. B. Ruderman. 1974. Glucose metabolism in rat skeletal muscle at rest. Effect of starvation, diabetes, ketone bodies and free fatty acids. Diabetes. 23:881-888.

27. Shonfeld, G., and D. Kipnis. 1968. Effects of fatty acids on carbohydrate and fatty acid metabolism of rat diaphragm. Am. J. Physiol. 215:513-522.

28. Rennie, M. J., and J. O. Holloszy. 1977. Inhibition of glucose uptake and glycogenolysis by availability of oleate in well-oxygenated perfused skeletal muscle. Biochem. J. 168:161-170.

29. Lassers, B., M. Wahlquist, L. Kaijser, and L. Carlson. 1972. Effect of nicotinic acid on myocardial metabolism in man at rest and during exercise. J. Appl. Physiol. 33:72-80.

30. Kaijser, L. 1980. Effect of metabolic intervention on substrate metabolism in the human heart. Adv. Myocardial. 2:51-59.

31. Kaijser, L., B. Lassers, M. Wahlquist, and L. Carlson. 1972. Myocardial lipid and carbohydrate metabolism in fasting men during prolonged exercise. J. Appl. Physiol. 32:847-858.

32. Rowe, J. W., J. B. Young, K. L. Miwakere, A. L. Stevens, J. Pallotta, and L. Landsberg. 1981. Effect of insulin and glucose infusions on sympathetic nervous system activity in normal man. Diabetes. 30:219-225.

33. Bergman, S. R., R. A. Lerch, K. A. A. Fox, P. A. Ludbrook, M. J. Welch, M. M. Ter-Pogossian, and B. E. Sobel. 1982. Temporal dependence of beneficial effects of coronary thrombolysis characterized by positron tomography. Am. J. Med. 73:573-581.

34. Marshall, R. C., J. H. Tillisch, M. E. Phelps, S. C. Huang, R. Carlson, E. Henze, and H. R. Schelbert. 1983. Identification and differentiation of resting myocardial ischemia and infarction in man with positron computed tomography, ${ }^{18} \mathrm{~F}$-labelled fluorodeoxyglucose and N-13 ammonia. Circulation. 67:766-778. 